\title{
EDUCAÇÃO EM SAÚDE ABORDANDO TEMA “DENGUE” DE FORMA INTERPROFISSIONAL: RELATO DE EXPERIÊNCIA.
}

\author{
HEALTH EDUCATION ADDRESSING THE THEME “DENGUE” IN AN \\ INTERPROFESSIONAL WAY: EXPERIENCE REPORT. \\ Cathiana Dalto, Maria Letícia Almeida Farias, Andressa Santos, Maira Motta, Magna Paris \\ Universidade Federal do Espírito Santo
}

\begin{abstract}
The report aims to report the experience in Health Education action with adults, discussing the dengue theme, guiding and raising awareness in communities in the city of São Mateus/ES. The intervention was prepared by the NN project, and was included in the activity calendar of two Family Health Strategy Units, entitled "D Day" (Mobilization Day related to Hypertension and Diabetes) with the objective of promoting education and disease prevention through interruption of the transmission chain. Inclusive techniques were used in order to promote community interaction in the exchange of knowledge. The population showed effective participation and interest, highlighting the cases notified and confirmed within the community. This triggered a greater reflection that led to a mobilization to clean the neighborhood. In this way, they contributed to social control and to the engagement of prevention and health promotion practices.
\end{abstract}

Keywords: Dengue. Health Education. Primary Health Care.

\section{Resumo}

$O$ relato tem o objetivo de relatar a experiência em ação de Educação em Saúde com adultos, discutindo o tema dengue, orientando $e$ conscientizando comunidades no município de São Mateus/ES. A intervenção foi elaborada pelo projeto NN, sendo incluída ao calendário de atividades de duas Unidades de Estratégia de Saúde da Família, intitulado "Dia D" (Dia de mobilização relacionado à Hipertensão e Diabete) com objetivo de promover educação e prevenção da doença por meio da interrupção da cadeia de transmissão. Foram utilizadas técnicas inclusivas de modo a promover a interação da comunidade na troca de saberes. A população demonstrou participação efetiva $e$ interesse, evidenciando os casos notificados e confirmados dentro da comunidade. Isso desencadeou uma maior reflexão que acarretou uma mobilização para a limpeza do bairro. Desta maneira, contribuíram no controle social e no engajamento de práticas de prevenção e promoção de saúde.

Palavras-chave: Dengue. Educação em Saúde. Atenção Primária à Saúde. 
Ao analisar os dados epidemiológicos dos últimos anos pode-se notar um aumento expressivo de doenças, especialmente as arboviroses, como a Dengue. Algumas ações humanas como o crescimento populacional urbano desordenado, geraram interferências e modificações dos ecossistemas, causando principalmente mudanças climáticas, a qual provavelmente é um dos fatores que contribuiu para o aumento dessas doenças ${ }^{(1)}$.

Observando o cenário atual, percebe-se que a "Dengue" é um tema popularizado dentro das comunidades, no entanto, com difícil mudança de comportamento dos indivíduos em relação à interrupção da cadeia de transmissão. A doença estabelece um fator preocupante para a saúde pública, já que mesmo sendo bastante discutida, inclusive pela mídia, ainda existe um aumento excessivo do número de casos. Nota-se também que grande parte dos criadouros infestados ou potenciais se encontra no interior dos domicílios, tendo as atividades educativas, alta representatividade no engajamento da população para a eliminação dos ambientes de proliferação e no esclarecimento sobre a dengue e sua etiologia ${ }^{(2)}$.

A educação em saúde é uma ferramenta conceituada como um processo educativo e inclusivo de formação à paciente, cuidadores e profissionais da área, de forma a estimular a construção de conhecimentos e autonomia aos cuidados individuais e coletivos, buscando melhor qualidade de vida ${ }^{(3)}$. O trabalho interprofissional na atenção primária integrado a práticas colaborativas é uma das melhores formas de enfrentar os desafios altamente complexos no setor de saúde, como as doenças recorrentes $^{(4)}$. Com isso, o trabalho interprofissional faz com que as ações de educação em saúde sejam extremamente ricas, pois, os profissionais conseguem transitar entre as áreas específicas de formação promovendo o ensino necessário ${ }^{(5)}$.

Para que essa educação seja eficaz é fundamental considerar a cultura popular, a prevalência da doença e a importância das ações movidas pela comunidade, promovendo a saúde através de diálogos entre os diversos saberes por meio do processo inclusivo, humanístico e participativo $^{(3)}$. Ao avaliar fator de prevalência no município de São Mateus/ES, território de atuação do projeto NN, notou-se um crescente aumento nos casos de dengue em 2019, chegando a 2321 notificações, tonando-se preocupante quando comparado ao ano anterior, onde houve apenas 40 notificações da doença ${ }^{(6)}$. Dessa forma, fez-se necessária à intervenção dos profissionais de saúde para promover uma discussão e compreensão da necessidade dos cuidados e prevenção contra a dengue.

Logo, o presente relato tem por objetivo descrever a atuação da equipe NN com profissionais da Atenção Primária ao criar ações propositivas sobre o problema, incluindo a população na prevenção da doença e na minimização dos agravos.

\section{Descrição da experiência}

Trata-se de uma Educação em Saúde voltada para adultos, desenvolvida em Unidades Básicas de Saúde (UBS) no município de São Mateus/ES, a qual foi associada ao evento instituído ao calendário de atividades das unidades, intitulado “Dia D" (Dia de mobilização relacionado à Hipertensão e Diabetes). Para a realização, utilizou-se a estratégia de "roda de conversa" sendo aplicados métodos de descontração, como alongamentos, apresentação pessoal e a entrega de balões coloridos, com o objetivo de promover maior participação e iniciativa por parte da comunidade presente. O propósito da ação era orientar e esclarecer a maior parte da população cadastrada no território da UBS sobre assuntos gerais da doença, com enfoque no conhecimento e interrupção da cadeia de transmissão do mosquito Aedes aegypti. Além disso, buscou-se identificar e esclarecer os sinais e sintomas da doença e a importância do atendimento na atenção primária.

Com o intuito de incluir a interprofissionalidade, discentes do curso de Enfermagem, Biologia e Farmácia, vinculados ao Projeto NN, atuaram como instrutores promovendo uma interligação entre sua área de formação com a doença, articulando as práticas colaborativas entre distintas profissões, facilitando assim a compreensão da comunidade através da integração de saberes. Por meio dessa articulação, o discente da biologia conseguiu informar com clareza sobre a morfologia do vetor e seu ciclo de vida, a importância da avaliação das potenciais localidades de proliferação do 
mosquito e a gravidade de não realizar vistorias nos domicílios. O estudante de enfermagem demonstrou os sinais e sintomas prevalentes na doença, e o discente de farmácia instruiu sobre quais medicamentos não devem ser utilizados e a importância de procurar uma UBS e realizar os exames laboratoriais durante a infecção. Além disso, o médico e a enfermeira presente complementaram as falas dos discentes, fazendo com o momento de conversa torna-se ainda mais proveitoso.

A intervenção abordou o conhecimento dos participantes através de um jogo de "mitos e verdades" sobre o tema apresentado, fazendo com que os indivíduos levantassem um balão vermelho quando a afirmação fosse considerada mito ou um balão verde quando a afirmação fosse verdadeira. Além disso, houve um momento de palavra aberta para que fossem compartilhadas experiências vividas com a doença, ou informações que ainda não haviam sido compartilhadas. Utilizou-se também um material explicativo na forma de fôlder, com finalidade de demonstrar a importância de realizar a prevenção contra o mosquito periodicamente, associado à interrupção do seu ciclo de vida. O fôlder dispunha de um calendário para anotar os dias de vistoria dentro dos domicílios e desenhos para tornar mais compreensíveis os principais locais que devem ser analisados. O tempo de execução da atividade foi em média de 35 minutos.

\section{Discussão}

Analisando a amplitude do tema e as várias formas utilizadas para combate a doença, estudos $^{(2)}$ demonstraram que a prática da Educação em Saúde realizada com o objetivo de reduzir criadouros do Aedes aegypti é mais efetiva do que a utilização de produtos químicos para o mesmo fim, sendo assim, considerada uma ótima prática para discutir temas recorrentes dentro da comunidade. Para melhor aproveitamento, notou-se que associar a educação em saúde a uma atividade do calendário da UBS é uma estratégia para incluir a temática da doença junto às atividades programadas, fazendo com que a educação em saúde ganhe destaque, substituindo as práticas campanhistas, além de estimular a participação da comunidade, incentivando um comportamento mais responsável pelo controle de potenciais criadouros ${ }^{(3)}$.
A metodologia utilizada por meio da roda de conversa foi positiva, uma vez que proporcionou liberdade de expressão aos participantes, na qual, os mesmos relataram suas experiências e opiniões sobre o assunto, além de sugestionarem ideias levando em consideração a realidade local e suas especificidades, demonstrando resultados semelhantes aos citados por Sampaio e colaboradores em $2014^{(7)}$. O jogo de mitos e verdades proporcionou a troca de informações de forma rápida e direta esclarecendo dúvidas da população e valorizando suas concepções. Logo, a forma que a atividade foi realizada abriu portas para uma multiplicação do conhecimento adquirido visando à orientação de vizinhos e familiares a respeito da prevenção contra a doença.

Segundo Pereira (2018), a interprofissionalidade na saúde e na educação articula novos arranjos de formação e produção dos elementos constitutivos do trabalho coletivo em saúde, além de agir como forma integral de saber e educar com pensamentos e ações integradas ${ }^{(8)}$. Para os discentes, as ações foram de extrema importância no seu desenvolvimento acadêmico, pois permitiu colocar em prática o aprendizado adquirido na da graduação associado à extensão do Projeto NN, demonstrando que a educação interprofissional contribui de forma positiva para os desafios de saúde, otimizando os serviços promovendo melhorias nos resultados ${ }^{(9)}$. Além disso, entrar em contato com a comunidade proporcionou novas experiências práticas enriquecidas pelo saber popular, transformandose em uma experiência única aos estudantes.

A avaliação da intervenção realizada pela equipe de saúde das UBS foi edificante. Posteriormente, houve relatos de agentes comunitárias sobre a melhora na limpeza dos bairros visualizada nas visitas domiciliares, além da preocupação da população com a interrupção do ciclo do mosquito. Esse tipo de relato demonstra que os problemas de saúde não se resolvem de forma independente, tendo grande responsabilidade os profissionais de saúde, os quais devem escutar as falas dos usuários e viceversa, oportunizando uma relação mais simétrica entre profissional da saúde e a comunidade ${ }^{(2)}$. Logo, o presente relato demonstra a importância de integrar a equipe interprofissional à educação em saúde na comunidade, promovendo uma troca de saberes que levam em conta os fatores locais, propiciando ações pontuais e com conteúdos próximos da realidade local, sem remover a autoridade da população. 


\section{Considerações finais}

A ação reforçou junto à população que os profissionais de saúde estão atentos as necessidades locais com o objetivo de promover saúde e prevenir doenças estimulando a participação dos indivíduos por meio do controle social, com o objetivo de melhorar a qualidade de vida da comunidade. A experiência levou à comunidade a repensar sobre as medidas de prevenção para a dengue por meio de uma abordagem diferente, leve e lúdica, considerando o conhecimento dos participantes sobre o tema, direcionando assim as abordagens. Socializar dados epidemiológicos da dengue com a comunidade e abordagens específicas do tema, fez com que despertasse uma preocupação evidente com a saúde própria, de familiares e vizinhos, promovendo mobilização no bairro para a limpeza e interrupção da cadeia de transmissão do vetor. Dessa forma a população contribuiu de forma positiva para o engajamento de práticas de prevenção e promoção de saúde na comunidade.

\section{Referências}

1. Martins MM, Prata-Barbosa A, Cunha AJLA da. Arboviral diseases in pediatrics. J Pediatr (Rio J) [Internet]. 2020;96(xx):2-11. Available from: https://doi.org/10.1016/j.jped.2019.08.005 2. Sales FMDS. Ações de educação em saúde para prevenção e controle da dengue: Um estudo em Icaraí, Caucaia, Ceará. Cienc e Saude Coletiva. 2008;13(1):175-84.

3. Maria E, Vasconcelos R De. 1095597077-1-Pb. 2015;41:27-34.

4. Farias DN de, Ribeiro KSQS, Anjos UU dos, Brito GEG de. Interdisciplinaridade E Interprofissionalidade $\mathrm{Na}$ Estratégia Saúde Da Família. Trab Educ e Saúde. 2017;16(1):141-62.

5. Da Silva JAM, Peduzzi M, Orchard C, Leonello VM. Interprofessional education and collaborative practice in primary health care. Rev da Esc Enferm. 2015;49(Speciallssue2):15-23.

6. TabNet Win32 3.0: DENGUE Notificações registradas no Sistema de Informação de Agravos de Notificação - Espírito Santo [Internet]. [cited 2021 Apr 21]. Available from:

http://tabnet.datasus.gov.br/cgi/deftohtm.exe?si nannet/cnv/denguebes.def

7. Sampaio J, Santos GC, Agostini M, Salvador A de S. Limites e potencialidades das rodas de conversa no cuidado em saúde: Uma experiência com jovens no sertão pernambucano. Interface Commun Heal Educ. 2014;18:1299-312.

8. Pereira MF. Interprofessionality and health: Connections and changing borders. Interface Commun Heal Educ. 2018;22:1753-6.

9. Araujo EC, Batista SSH, Gerab IF, li B, Cariri R, li CDA, et al. Marco para Ação em Educação Interprofissional e Prática Colaborativa. Cad Saude Publica [Internet]. 2010;377(1):62p. Available

from:http://www.fen.ufg.br/revista/v14/n1/v14n 1a10.htm.\%5Cnhttp://www.who.int/hrh/nursing _midwifery/en/

\section{Endereço para Correspondência}

Cathiana do Carmo Dalto Banhos

Universidade Federal do Espírito Santo, Campus CEUNES - Departamento deCiências da Saúde. Rodovia Governador Mário Covas, Km 60, Bairro Litorâneo, São Mateus, ES, Brasil.

CEP: $29932-540$

E-mail: cathianadalto@yahoo.com

Recebido em 18/11/2020

Aprovado em 10/12/2021

Publicado em 30/12/2021 